Background Stent retriever (SR) thrombectomy is commonly used for the treatment of emergent large vessel occlusion (ELVO) in acute ischemic stroke (AIS). Clot imaging parameters such as clot length, diameter, distance to the internal carotid artery (ICA) terminus and vessel angle where the SR is deployed may predict the likelihood of achieving first-pass effect (FPE). Most of the proposed factors that seem to affect recanalization success have been studied individually, and conflicting data derived from clinical versus in vitro studies using 3D printed models of intracranial circulation currently exist.

Methods Using patient-specific 3D phantoms of the cervical and intracranial circulation, we simulated MCA M1 and M2 occlusions treated with SR thrombectomy using Solitaire (Medtronic) or Trevo (Styker). Our primary outcome was first-pass effect, defined as Thrombolysis in Cerebral Infarction score of 2c-3 achieved after a single thrombectomy attempt. We also performed retrospective analysis of same clot imaging characteristics of consecutive cases of MCA occlusion and its association with FPE matching the $3 \mathrm{D}$ in vitro experiments. Analysis was conducted using IBM SPSS Statistics Version 25 (IBM Corp., Armonk, NY). Chi-square tests, and bivariate logistic regressions were the main statistical tests used in analysis. A p-value of less than 0.05 was considered to indicate statistical significance. 95\% confidence intervals (95CI) were generated.

Results We compared 42 thrombectomy experiments performed using patient-specific $3 \mathrm{D}$ in vitro models with a retrospective cohort of 42 patients treated with SR thrombectomy. We found that in the in vitro cohort, higher MCA angulation was associated with a lower likelihood of FPE (Odds ratio $(\mathrm{OR})=0.967$ 95CI $=0.944-0.991, \mathrm{p}=0.008$ ). Meanwhile in the in vivo cohort, higher MCA angulation was associated with a higher likelihood of FPE (OR $=1.039$ 95CI $=1.003$ 1.077, $\mathrm{p}=0.033)$. Neither clot length nor location of clot (M1 vs M2) was associated with a difference in FPE rates in either cohort.

Discussion Comparison of SR thrombectomy performed during actual MCA occlusion cases versus patient specific 3D replicas revealed MCA angulation as an independent predictor of procedure success or failure. However, the opposite direction of effect was observed between the two studied environments, indicating potential limitations of studying SR thrombectomy using 3D models of LVO.

Disclosures M. Mokin: 1; C; NIH R21NS109575. E. Pressman: None. K. Sommer: None. C. Ionita: None.

\section{E-127 COMBINED MIVI-QTM ASPIRATION CATHETER-STENT RETRIEVER APPROACH TO STROKE THROMBECTOMY: PRELIMINARY CLINICAL EXPERIENCE}

S Remollo*. Interventional Neuroradiology, Hospital Universitari Germans Trias i Pujol, Badalona, Spain

\subsection{6/neurintsurg-2021-SNIS.222}

Background Large-bore aspiration catheters enabling greater flow rates and suction force for mechanical thrombectomy might improve outcomes in patients with stroke secondary to large-vessel occlusion. Complete or near-complete recanalization after a single thrombectomy pass (first-pass effect) is associated with improved clinical outcomes. We assessed the efficacy and safety of novel MIVI $\mathrm{Q}^{\mathrm{TM}}$ aspiration catheters in combination with stent-retriever devices.
Methods We retrospectively analyzed demographics, procedure characteristics, and clinical data from consecutive patients with acute anterior large-vessel occlusion treated with a combined approach using MIVI $\mathrm{Q}^{\mathrm{TM}}$ aspiration catheters and stent retrievers. Reperfusion was defined according to the Modified Thrombosis in Cerebral Infarction (mTICI) score. Clinical outcomes were measured by the National Institute of Health Stroke Scale (NIHSS) and modified Rankin Scale (mRS) scores.

Results We included 52 patients [median age, 75 y (IQR:64$83) ; 31$ (59.6\%) women; 14 (26.9\%) with occlusions in the terminal internal carotid artery, $26(50 \%)$ in middle cerebral artery (MCA) segment M1, and 12 (23.1\%) in MCA segment M2; median NIHSS score at admission, 19 (IQR:13-22)]. After the first pass, $25(48 \%)$ patients had $m T I C I \geq 2$ c. At the

\begin{tabular}{ll}
$\begin{array}{l}\text { Abstract E-127 Table } 1 \\
\text { efficacy }\end{array}$ & Angiographic outcomes and clinical \\
\hline Groin-puncture-to-revascularization [min], median (IQR) & $42(22-66)$ \\
\hline$Q^{T M}$ tFPE, $n(\%)$ & $22(42.3)$ \\
$Q^{T M}$ FPE, n (\%) & $25(48)$ \\
mTICI 3, n (\%) & $28(53.8)$ \\
mTICI 2c-3, n (\%) & $35(67.3)$ \\
mTICI $\geq 2 b, n(\%)$ & $47(90.4)$ \\
$m R S ~ 0-2$ at 90 days, n (\%) & $24(46.2)$ \\
sICH, n (\%) & $1(1.9)$ \\
Serious device-related adverse events, $n(\%)$ & $0(0)$ \\
Serious procedure related adverse events, $n(\%)$ & $1(1.9)$ \\
\hline
\end{tabular}

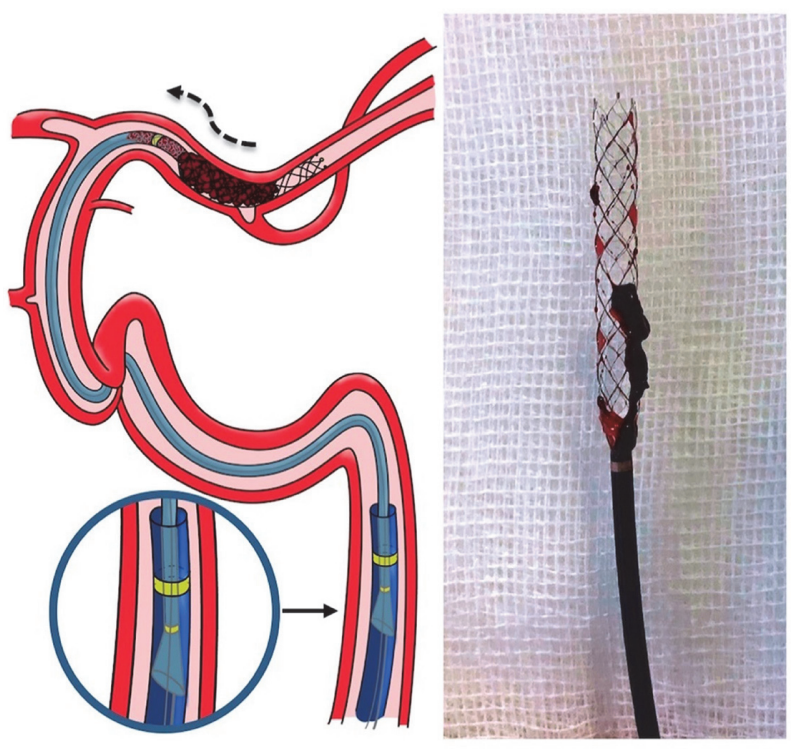

Abstract E-127 Figure 1 LEFT Illustration of the $Q^{\mathrm{TM}}$ and stent retriever combined approach during the thrombus wedging maneuver. After removing the microcatheter, the stent retriever has been slightly retracted and the $Q^{\mathrm{TM}}$ catheter carefully advanced under pump aspiration to ensure better engagement of the thrombus. The flared proximal tip of the $Q^{T M}$ catheter (magnified inset) ensures a tight seal between its outer surface and the Super 90 guiding catheter's inner surface, enabling increased suction power (curved black dashed arrow) directly through the guide catheter. RIGHT Q6 aspiration catheter and Stent Retriever with a "pinned" thrombus 
end of the procedure, $47(90.4 \%)$ had $\mathrm{mTICI} \geq 2 \mathrm{~b}$ and 35 $(67.3 \%)$ had $\mathrm{mTICI} \geq 2 \mathrm{c}$. No serious device-related adverse events were observed. Symptomatic intracranial hemorrhage developed in $1(1.92 \%)$ patient. Mean NIHSS score was 13 at 24 hours and 5 at discharge. At 90 days, 24 (46.2\%) patients were functionally independent (mRS 0-2).

Conclusions This preliminary study found good efficacy and safety for MIVI $\mathrm{Q}^{\mathrm{TM}}$ aspiration catheters used in combination with stent-retriever devices.

Table Shows main Angiographic, Clinical efficacy and safety outcomes.

Disclosures S. Remollo: None.

\section{E-128 COMPARISON OF EFFICACY AND OUTCOMES BETWEEN DIFFERENT THROMBECTOMY TECHNIQUES IN ACUTE BASILAR ARTERY OCCLUSION: A DUAL CENTER EXPERIENCE}

${ }^{1} \mathrm{~A}$ Monteiro* ${ }^{1} \mathrm{G}$ Cortez, ${ }^{1} \mathrm{M}$ Wagas, ${ }^{1} \mathrm{H}$ Rai, ${ }^{1} \mathrm{~A}$ Baig ${ }^{1} \mathrm{R}$ Dossani, ${ }^{1} \mathrm{C}$ Cappuzzo, ${ }^{1} \mathrm{~F}$ Almayman, ${ }^{2} \mathrm{~A}$ Aghaebrahim, ${ }^{1} \mathrm{~J}$ Davies, ${ }^{2} \mathrm{E}$ Sauvageau, ${ }^{1} \mathrm{~K}$ Snyder, ${ }^{2} \mathrm{R}$ Hanel, ${ }^{1} \mathrm{E}$ Levy, ${ }^{1}$ A Siddiqui. 'Neurosurgery, University at Buffalo Neurosurgery, Buffalo, NY; ${ }^{2}$ Cerebrovascular and Endovascular Surgery, Baptist Neurological Institute and Lyerly, Jacksonville, $F$

\subsection{6/neurintsurg-2021-SNIS.223}

Introduction Acute basilar artery occlusion $(\mathrm{aBAO})$ is a rare large vessel occlusion (LVO) associated with high morbidity and mortality. Modern thrombectomy devices are highly effective in achieving recanalization, but direct comparison of different techniques have not been performed in aBAO yet.

Methods Retrospective analysis of databases of LVO treated with endovascular intervention from 2 US endovascular neurosurgery centers. We included patients $\geq 18$-year-old with acute basilar artery occlusion treated with stent-retrievers or largebore aspiration catheters. The efficacy of Solumbra, Direct Aspiration First Pass (ADAPT) and stent-retriever alone (SR) techniques were compared, in addition to clinical outcomes.

Results Eighty-three patients were included. Median age was 67 years (IQR, 58-76) and 39.8\% were female. Median NIHSS at admission was 16 (IQR, 10-21). Intravenous alteplase was administered to 26 patients (31.3\%). Median time from onset-of-symptoms to puncture was 256 minutes (IQR, 157.5-363.0). Solumbra had significant higher rate of modified First Pass Effect (mFPE; $55 \%$ vs $31.8 \%, \mathbf{P}=0.032$ ), but not true First Pass Effect (FPE; 45\% vs 34.9\%, P=0.346). Good outcome (mRS 0-2) was not significantly different between the 3 techniques. Poor outcome (mRS 4-6) was associated with a higher median NIHSS (12.5 vs $19, \mathrm{P}=0.007)$, lower mean number of passes $(1.92$ vs $1.82, \mathrm{P}<0.001)$, higher rate of adjunctive therapy use $(10 \%$ vs $0 \%, \mathbf{P}<0.001)$ and higher rate of intraoperative complications $(10.7 \%$ vs $14.5 \%, \mathrm{P}=\mathbf{0 . 0 0 6})$. NIHSS at admission was found to significantly predict good outcome (OR 0.98, 95\%CI 0.97-.099, P=0.032). Incomplete recanalization after thrombectomy significantly predicted mortality (OR 1.68, 95\% CI 1.18-2.39; P=0.005).

Conclusion The evaluated techniques resulted in high recanalization rate. Solumbra is associated with higher rate of mFPE than ADAPT and SR, but clinical outcomes were similar. A lower NIHSS predicted patients with better prognosis, while incomplete recanalization after thrombectomy predicted mortality. Modern thrombectomy techniques are technically efficient in aBAO, but more studies are needed on prognostic factors of these patients.
Disclosures A. Monteiro: None. G. Cortez: None. M. Waqas: None. H. Rai: None. A. Baig: None. R. Dossani: None. J. Cappuzzo: None. F. Almayman: None. A. Aghaebrahim: None. J. Davies: 1; C; National Center for Advancing Translational Sciences of the National Institutes of Health under award number KL2TR001413 to the University at Buffalo. 2; C; Medtronic. 4; C; Cerebrotech, RIST Neurovascular. E. Sauvageau: None. K. Snyder: 2; C; Canon Medical Systems Corporation, Penumbra Inc., Medtronic, and Jacobs Institute. R. Hanel: None. E. Levy: 2; C; Claret Medical, GLG Consulting, Guidepoint Global, Imperative Care, Medtronic, Rebound, StimMed;. 4; C; NeXtGen Biologics, RAPID Medical, Claret Medical, Cognition Medical, Imperative Care (formerly the Stroke Project), Rebound Therapeutics, StimMed, Three Rivers Medical;. A. Siddiqui: 2; C; Amnis Therapeutics, Boston Scientific, Canon Medical Systems USA Inc., Cerebrotech Medical Systems Inc., Cerenovus, Corindus Inc., Endostream Medical Ltd., Imperative Care, Inc. Integra LifeSciences C. 4; C; Adona Medical, Inc, Amnis Therapeutics, (Purchased by Boston Scientific October 2017), Blink TBI Inc., Buffalo Technology Partners Inc., Cerebrotech Medical Systems, Inc., Cognition Medical, Endostrea.

\section{E-129 FLOW REDIRECTION ENDOLUMINAL DEVICE (FRED) FOR TREATMENT OF INTRACRANIAL ANEURYSMS: A SYSTEMATIC REVIEW}

${ }^{1} \mathrm{M}$ Waqas*, ${ }^{1} \mathrm{R}$ Dossani, ${ }^{2} \mathrm{M}$ Alkhaldi, ${ }^{3} \mathrm{~J}$ Neveu, ${ }^{1} \mathrm{~J}$ Cappuzzo, ${ }^{1} \mathrm{~J}$ Liam, ${ }^{1} \mathrm{~A}$ Khan, ${ }^{3} \mathrm{~V}$ Lazarov, ${ }^{1} \mathrm{~A}$ Monteiro, ${ }^{1} \mathrm{~J}$ Davies, ${ }^{1} \mathrm{~A}$ Siddiqui, ${ }^{1} \mathrm{E}$ Levy. ${ }^{1}$ Neurosurgery, University at Buffalo Neurosurgery, Buffalo, NY; ${ }^{2}$ Neurosurgery, Imam Abdulrhman Bin Faisal Univeristy, Dammam, Saudi Arabia; ${ }^{3}$ Neurosurgery, Jacobs School of Medicine and Biomedical Sciences, Buffalo, NY

\subsection{6/neurintsurg-2021-SNIS.224}

Introduction The Flow Redirection Endoluminal Device (FRED; MicroVention) is a dual-layered flow diverter used for the treatment of intracranial aneurysms. The objective of this systematic review was to compile device-related safety and effectiveness data.

Methods The literature from January 1, 2013 to June 30, 2020 was searched for studies describing use of the FRED for intracranial aneurysm treatment irrespective of aneurysm location and morphology. The review included anterior and posterior circulation ruptured and unruptured saccular, fusiform or dissection, and blister aneurysms. MeSH terms related to "flow re-direction endoluminal device" and "FRED for aneurysms" were used. Data related to indication, complications, and rates of aneurysm occlusion were retrieved and analyzed.

Results Twenty studies with 1674 intracranial aneurysms were included in this review. The overall reported morbidity was 0$20 \%$. Overall procedure-related mortality was $1.6 \%$ (range 0 $6 \%$ ). Complication rates fell into 5 categories: technical (3.8\%), ischemic (3.9\%), thrombotic orstenotic (5.9\%), hemorrhagic $(1.5 \%)$, and non-neurological $(0.8 \%)$. The aneurysm occlusion rate between 0 and 3 months (reported in 9 studies) was $47.8 \%$. The occlusion rate between 4 and 6 months (reported in 11 studies) was $73.1 \%$. Occlusion rates continued to increase to $75.1 \%$ at7-12 months (reported in 9 studies) and $87.0 \%$ for follow up beyond 1 year (reported in 8 studies).

Conclusion This review indicated that the FRED is a safe and effective for the treatment of intracranial aneurysms. Future studies should directly compare the FRED with other flow 